\title{
On Self-Similarity of Top Production at Tevatron
}

\author{
Mikhail Tokarev $^{1^{*}}$, Imrich Zborovský ${ }^{2}$ \\ ${ }^{1}$ Joint Institute for Nuclear Research, Moscow, Russia \\ ${ }^{2}$ Nuclear Physics Institute, Academy of Sciences of the Czech Republic, Prague, Czech Republic \\ Email: "tokarev@jinr.ru
}

Received June 7, 2012; revised July 1, 2012; accepted July 31, 2012

\begin{abstract}
This paper presents the results of analysis of the DØ $1.0 \mathrm{fb}^{-1}$ data on top-quark differential cross section measurements at the Fermilab Tevatron collider at $\sqrt{s}=1960 \mathrm{GeV}$ in the framework of $z$-scaling approach. The flavor independence of scaling function $\Psi(z)$ observed in $p p$ and $p \bar{p}$ interactions over a wide collision energy range $\sqrt{s}=19$ $1960 \mathrm{GeV}$ has been verified. This property of $\Psi(z)$ was found for different hadrons - from $\pi$-mesons up to $Y$ particles. The flavor independence of $\Psi(z)$ is used as indication on self-similarity of the top-quark production. A tendency to saturation of $\Psi(z)$ at low $z$ for top-quark production has been confirmed. Momentum fraction $x_{1}$ of the incoming (anti)protons as a function of the scaled transverse momentum $p_{T} / m$ and masses of heavy mesons is studied. We anticipate that the data on low- and high- $p_{T}$ inclusive spectra of the top-quark production at the Tevatron and LHC energies could be of interest to verify self-similarity over a wide range of masses and different flavor content of produced particles.
\end{abstract}

Keywords: Inelastic Cross Section; Proton-AntiProton Collisions; Top Production; Scaling; Self-Similarity

\section{Introduction}

The measurements of the top-quark transverse momentum distribution have been performed at the Fermilab Tevatron collider at $\sqrt{s}=1800$ and $1960 \mathrm{GeV}$ by the CDF [1] and DØ [2] Collaborations, respectively. The integrated luminosities of CDF and DØ data samples are $106 \mathrm{pb}^{-1}$ and $1 \mathrm{fb}^{-1}$. The top-quark is the heaviest known elementary particle and was discovered at the Tevatron $p \bar{p}$ collider in 1995 by the CDF and DØ Collaborations $[3,4]$ at a mass of around $170 \mathrm{GeV}$. It is expected [5-12] that the top physics is extremely important for scientific search for new phenomena.

In the given paper we have analyzed the $\mathrm{D} \varnothing$ data using the method known as $z$-scaling $[13,14]$. Main features of the approach in $p p$ and $p \bar{p}$ interactions at FNAL, CERN, and BNL (RHIC) energies were presented and discussed in $[15,16]$. Some results of analysis of the LHC data on the charged hadron [17], $K_{S}^{0}$-meson [18], and jet $[19,20]$ production are presented in [21-23]. The method allows us to perform systematic analysis of data on inclusive cross sections of hadrons, direct photons, and jets under different kinematic conditions. Scaling function $\Psi(z)$ and scaling variable $z$ are expressed via experimentally measurable quantities: inclusive and total inelastic cross sections, multiplicity density, momenta and masses of colliding and produced particles and using some physical parameters. The shape of the scaling function was found to be independent of the collision energy, multiplicity density of particles, detection angle and hadron type. The power behavior of function $\Psi(z) \sim z^{-\beta}$ was established in high- $z$ (high- $p_{T}$ ) range. At low $z$ (low$p_{T}$ ), saturation of the scaling function was found down to a value of $z \approx 10^{-3}[16,22]$. It has been concluded that $z$-scaling reflects self-similarity of the hadron structure, constituent interactions and hadronization process. The analyzed experimental data cover a wide range of the collision energies, transverse momenta and angles of the produced particles. The energy, angular and multiplicity independence of scaling function $\Psi(z)$ gives strong constraints on the values of parameters $\delta, c$, and $\varepsilon$ entering in definition of $z$. The parameter $c$ which controls the behavior of $\Psi(z)$ at low $z$ has analogy with the "specific heat" of the produced medium associated with the inclusive particle production. The scaling in $p p$ and $p \bar{p}$ collisions is consistent with the constant value of $c=0.25$. A possible change of this parameter could be assumed to be an indication of the phase transition of the matter produced in high energy collisions. The structure of the interacting objects produced at high momenta is characterized by parameter $\delta$ interpreted as a nucleon fractal dimension. The scaling is consistent with the constant value of $\delta=0.5$ for all types of the analyzed inclusive 
hadrons. The fragmentation process is parameterized in terms of dimension $\varepsilon_{F}$ which increases together with the hadron mass.

In this paper we have analyzed the data [2] on transverse momentum spectra of the top-quark production in $p \bar{p}$ collisions at energy $\sqrt{s}=1960 \mathrm{GeV}$ in the middle rapidity range obtained by the $\mathrm{D} \emptyset$ Collaboration at the Tevatron. The measurements select the events with an isolated lepton having transverse momentum $p_{T}$ of at least $20 \mathrm{GeV} / \mathrm{c}$ and a pseudo-rapidity of $|\eta|<1.1(e+$ jets $)$ or $|\eta|<2.0(\mu+j e t s)$. A cut on the missing transverse energy of $20 \mathrm{GeV}$ was applied. Furthermore at least four jets were required with $p_{T}>20 \mathrm{GeV} / \mathrm{c}$ and $|\eta|<2.5$, an additional cut of $p_{T}>40 \mathrm{GeV} / \mathrm{c}$ was applied for the leading jet. Finally at least one jet needs to be identified as a $b$-jet. Additional constraints are used to reconstruct the event kinematics: the masses of two $W$ bosons are constrained to $80.4 \mathrm{GeV}$. The masses of the two reconstructed top quarks are assumed to be equal.

The results of analysis of the top inclusive cross sections are compared with Tevatron data [24-26] on $J / \psi, D^{0}$, $B$, and $Y$ particle spectra at $\sqrt{s}=1960$ and $1800 \mathrm{GeV}$ in the $z$-presentation. We have verified the flavor independence of $\Psi(z)$ including the inclusive top-quark measurements in this region. A microscopic scenario of hadron production in the $z$-scaling approach is used to estimate the energy loss and recoil mass at a constituent level in the dependence on transverse momentum of an inclusive particle. This gives the specific dependence of the momentum fraction $x_{1}$ characteristic for different types of produced hadrons. The $p_{T}$-behavior of the fraction $x_{1}$ for the top-quark production is compared with other particles.

We expect that systematic measurements of the inclusive differential spectra of the top-quark as a function of the transverse momentum at LHC energies could give new information on self-similarity of the heavy flavor production in the super high energy domain.

\section{2. $z$-Scaling}

Here we follow the basic ideas of the $z$-scaling approach $[15,16]$. It is assumed that the collision of extended objects (hadrons, nuclei) at sufficiently high energies could be considered to be an ensemble of individual interactions of their constituents (partons, quarks, gluons). Structures of the colliding objects are characterized by parameters $\delta_{1}$ and $\delta_{2}$. The constituents of the incoming objects (hadrons or nuclei) with masses $M_{1}, M_{2}$ carry away fractions $x_{1}, x_{2}$ of their momenta $P_{1}, P_{2}$. The inclusive particle has a fraction (denoted by $y_{b}$ ) of the momentum of the object produced in the constituent collision in the observed direction. Its fragmentation is characterized by parameter $\epsilon_{a}$. The fragmentation in the recoil direction is described by parameter $\epsilon_{b}$ and momentum fraction $y_{b}$. Multiple interactions of the constituents are considered to be similar. This property reflects the self-similarity of the hadron interactions at the constituent level.

\subsection{Momentum Fractions $x_{1}, x_{2}, y_{a}$ and $y_{b}$}

The elementary sub-process is considered to be a binary collision of the constituents with masses $x_{1} M_{1}$ and $x_{2} M_{2}$ resulting in the scattered and recoil objects with masses $m_{1} / y_{a}$ and $x_{1} M_{1}+x_{2} M_{2}+m_{2} / y_{b}$ in the final state. The produced secondary objects transform into real particles after the constituent collisions. The registered particle with mass $m_{1}$ and 4-momentum $p$ is produced with its hadron counterpart with mass $m_{2}$ carrying the momentum fractions of the produced recoil. The momentum conservation law of the constituent sub-process is written in the following form:

$$
\left(x_{1} P_{1}+x_{2} P_{2}-p / y_{a}\right)^{2}=M_{X}^{2} .
$$

Here $M_{X}$ is the recoil mass and

$$
M_{X}=x_{1} M_{1}+x_{2} M_{2}+m_{2} / y_{b} .
$$

The production of the associated particle with mass $m_{2}$ ensures conservation of the additive quantum numbers. Equation (1) is an expression of the locality of the hadron interaction at a constituent level. It represents a kinematic constraint on momentum fractions $x_{1}, x_{2}, y_{a}$, and $y_{b}$ which determine the underlying elementary sub-process.

The structure of colliding objects and fragmentation of the systems formed in scattered and recoil directions are characterized by parameters $\delta_{1}, \delta_{2}$, and $\epsilon_{a}, \epsilon_{b}$, respectively. The parameters are related with the corresponding momentum fractions by function

$$
\Omega=\left(1-x_{1}\right)^{\delta_{1}}\left(1-x_{2}\right)^{\delta_{2}}\left(1-y_{a}\right)^{\varepsilon_{a}}\left(1-y_{b}\right)^{\varepsilon_{b}} .
$$

Quantity $\Omega$ is proportional to the relative number of all constituent configurations in the inclusive reaction, which contain the configuration defined by fractions $x_{1}, x_{2}, y_{a}$, and $y_{b}$. The $\Omega$ is interpreted as a relative volume which occupies these configurations in the space of the momentum fractions. Parameters $\delta_{1}, \delta_{2}$, and $\epsilon_{a}, \epsilon_{b}$ are taken as fractal dimensions in the parts of the space of the momentum fractions which correspond to the colliding objects and fragmentation processes, respectively. For the given values of $\delta_{1}, \delta_{2}$, and $\epsilon_{a}, \epsilon_{b}$ the fractions $x_{1}, x_{2}, y_{a}$, and $y_{b}$ are determined in such a way to maximize the function $\Omega$, simultaneously fulfilling condition (1).

In the case of $p p(p \bar{p})$ interactions we have $M_{1}=M_{2}=m_{N}$ and set $\delta_{1}=\delta_{2} \equiv \delta$. It is assumed that the fragmentation of the objects moving in the scattered and recoil directions can be described by the same parameter $\epsilon_{a}=\epsilon_{b}=\varepsilon_{F}$ which depends on the type of the inclusive particle. The values of parameters $\delta$ and $\varepsilon_{F}$ 
are determined according to the self-similarity requirements of experimental data in $z$-presentation. They were found to have constant values in $p p$ and $p \bar{p}$ collisions at high energies.

\subsection{Scaling Variable $z$ and Scaling Function $\Psi(z)$}

The self-similarity of hadron interactions reflects the property that hadron constituents and their interactions are similar. The self-similarity variable $z$ is defined as follows:

$$
z=z_{0} \Omega^{-1},
$$

where $z_{0}=\sqrt{s_{T}} /\left(\mathrm{d} N_{c h} /\left.\mathrm{d} \eta\right|_{0}\right)^{c} m_{N}$, and $\Omega$ is the maximal value of (2) with condition (1). For the above inclusive reaction the quantity $z$ is proportional to the transverse kinetic energy $\sqrt{s_{T}}$ of the constituent subprocess consumed for the production of the inclusive particle and its counterpart with masses $m_{1}$ and $m_{2}$, respectively. The quantity $\mathrm{d} N_{c h} /\left.\mathrm{d} \eta\right|_{0}$ is the corresponding multiplicity density of charged particles produced in the central region of the reaction at the pseudo-rapidity $\eta=0$. Parameter $c$ characterizes properties of the produced medium. It is interpreted as "specific heat". The constant $m_{N}$ is taken to be a nucleon mass.

Scaling function $\Psi(z)$ is expressed in terms of the experimentally measured inclusive cross section $E \mathrm{~d}^{3} \sigma / \mathrm{d} p^{3}$, the multiplicity density $\mathrm{d} N / \mathrm{d} \eta$, and the total inelastic cross section $\sigma_{\text {inel }}$ as follows [15]:

$$
\Psi(z)=-\frac{\pi s}{(\mathrm{~d} N / \mathrm{d} \eta) \sigma_{\text {inel }}} J^{-1} E \frac{\mathrm{d}^{3} \sigma}{\mathrm{d} p^{3}} .
$$

Here $s$ is the square of the center-of-mass energy and $J$ is the corresponding Jacobian. The multiplicity density $\mathrm{d} N / \mathrm{d} \eta$ in (4) depends on the center-of-mass energy, centrality, and on the production angles at which the inclusive spectra were measured. The above expression can be rewritten in the central interaction region into the following form:

$$
\Psi(z)=-\frac{1}{N \sigma_{\text {inel }}}\left(\frac{\mathrm{d} z}{\mathrm{~d} p_{T}}\right)^{-1} \frac{\mathrm{d} \sigma}{\mathrm{d} p_{T}} .
$$

The scaling function is normalized as follows:

$$
\int_{0}^{\infty} \Psi(z) \mathrm{d} z=1
$$

It allows us to interpret $\Psi(z)$ as a probability density of the production of the inclusive particle with the corresponding value of variable $z$.

\section{Flavor Independence of $\Psi(z)$ and Self-Similarity of Top Production}

The flavor independence of hadron production means that spectra of particles with a different flavor content can be described by universal scaling function $\Psi(z)$ in $z$-presentation $[15,16]$. Our previous analysis is based on the observation that simultaneous energy, angular and multiplicity independence of the $z$-scaling for negative pions, kaons, and anti-protons produced in proton-proton collisions gives the same shape of the scaling function. The flavor independence of $\Psi(z)$ was also confirmed for other inclusive particles including the heavy quarkonia, $J / \psi$ [24], and $Y$ [26], measured at the Tevatron energies $\sqrt{S}=1960$ and $1800 \mathrm{GeV}$. The property of $\Psi(z)$ was observed at very small values of $z \approx 10^{-3}$. In the region $z \leq 0.1$ we observe a saturation of scaling function $\Psi(z)$ which can be approximated by a constant.

In this paper we analyze the data [2] on the differential cross section of the top-quark production measured by the DØ Collaboration at the Tevatron as a function of the transverse momentum $p_{T}$ at a middle rapidity. We have shown that the flavor independence of the $z$-presentation of hadron spectra is valid for the top-quark production as well. We exploit the scaling transformation

$$
z \rightarrow \alpha_{F} \cdot z, \Psi \rightarrow \alpha_{F}^{-1} \cdot \Psi
$$

to compare the shape of scaling function $\Psi(z)$ for different hadron species. Parameter $\alpha_{F}$ is the scale independent quantity. The transformation does not destroy the shape of $\Psi(z)$. It preserves the normalization Equation (6) and the energy, angular and multiplicity independences of the $z$-presentation of particle spectra.

Figure 1(a) shows the $z$-presentation of the spectra of heavy hadrons $\left(J / \psi, D^{0}, B\right.$, and $\left.Y\right)$ [24-26] obtained in $p \bar{p}$ collisions at the Tevatron energies $\sqrt{s}=1960$ and $1800 \mathrm{GeV}$ in the central rapidity region. The experimental data are shown by symbols. The data include measurements up to small transverse momenta $\left(p_{T} \approx 125\right.$ $\mathrm{MeV} / \mathrm{c}$ for charmonia, $p_{T} \approx 290 \mathrm{MeV} / \mathrm{c}$ for bottomia, and $p_{T} \approx 500 \mathrm{MeV} / \mathrm{c}$ for $B$-mesons). The data on the $\pi^{-}$-meson spectra at $\sqrt{s}=53 \mathrm{GeV}$ [27] are used as reference data. As seen from Figure 1(a) the shape of scaling function is the same for hadrons with light and heavy flavors produced in $p p$ and $p \bar{p}$ collisions in the range $z=0.001-4$. This is indicated by the solid line. One can see that distributions of different hadrons are sufficiently well described with a single curve over a wide $z$-range $(0.001-10)$. Function $\Psi(z)$ changes more than by six orders of magnitude in this region. The values of parameters $\alpha_{F}$ and $\varepsilon_{F}$ shown in this Figure are consistent with the energy, angular and multiplicity independences of the $z$-presentation of the spectra for different hadrons. The parameters were found to be independent of kinematic variables $\left(\sqrt{s}, p_{T}\right.$, and $\left.\theta_{c m s}\right)$. The scale factors $\alpha_{F}$ are constants. It allows us to describe $z$-presentation of the spectra for different hadron species 
with a single function. The collapse of data points onto a single curve corresponds to the estimated errors of $\alpha_{F}$ at the level of $20 \%$.

Figure 1(b) demonstrates the results of analysis of the Tevatron data [2] on the top-quark spectra measured by the DØ Collaboration in $p \bar{p}$ collisions at the energy $\sqrt{s}=1960 \mathrm{GeV}$ and the central rapidity range in $z$ presentation. The solid line is the same curve as depicted in Figure 1(a). Scaling function $\Psi(z)$ for the topquark distribution was calculated according to Equation (5). The vertical errors are given by a quadratic sum of the statistical uncertainties and the systematic uncertainties on the shape of the cross section in each $p_{T}$-bin. The

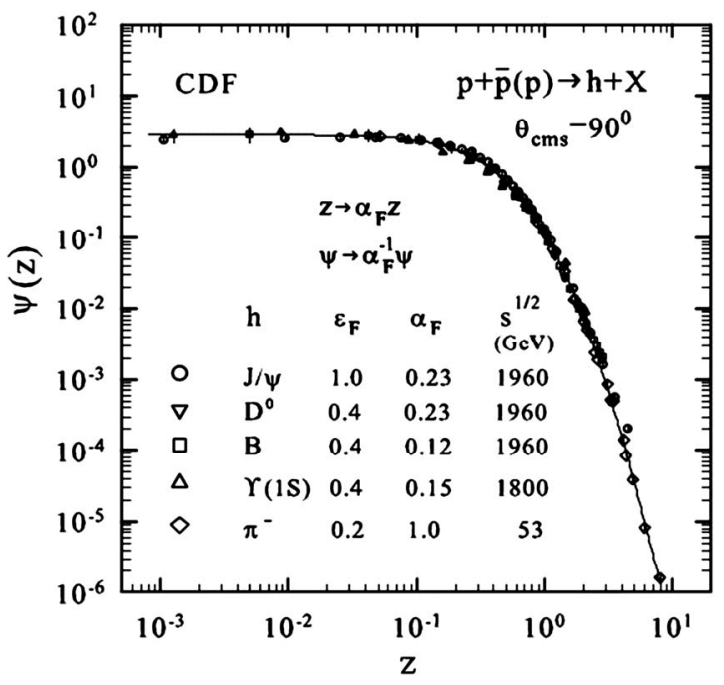

(a)

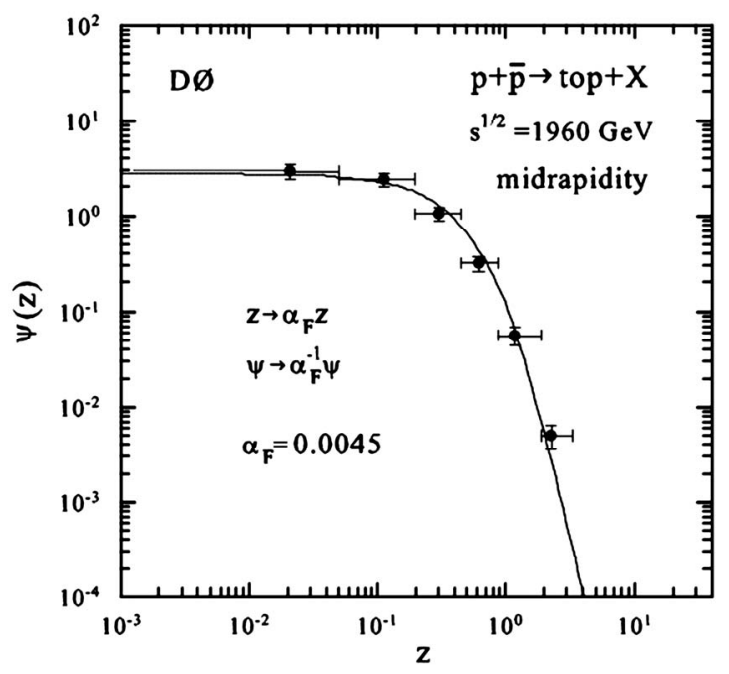

(b)

Figure 1. The flavor independence of $z$-scaling. The spectra of (a) $J / \psi, D^{\mathbf{0}}, B, Y$ mesons; and (b) top-quarks produced in $p \bar{p}$ collisions at the Tevatron in $z$-presentation. The spectra of $\pi^{-}$mesons were measured in $p p$ collisions at ISR. The data are taken from [2,24-27]. The solid line in (b) is the same as in (a). horizontal errors refer to the width of the bins. The condition (6) is satisfied with the normalization $N \sigma_{\text {inel }}=n \sigma_{\bar{t} \bar{t}}$ and $n=2$. This corresponds to two entries per event with the total normalization to the $t \bar{t}$ production cross section $\sigma_{t \bar{t}}=8.31 \mathrm{pb}[2]$.

The values of the fractal dimension $\delta=0.5$ and "specific heat" $c=0.25$ are the same as used in the previous analyses of the inclusive spectra $[15,16,21]$. We have set $\varepsilon_{\text {top }}=0$ in the case of the top-quark since no energy loss is assumed in the elementary $t \bar{t}$ production process. This choice corresponds to $y_{a}=y_{b}=1$ in the whole $p_{T}$-range. The value of $\alpha_{F}$ in the transformation (7) is found to be $\alpha_{t o p} \approx 0.0045$. No additional parameters were used.

As it is seen from Figure 1(b), the $z$-presentation of the top-quark transverse momentum distribution follows the shape of the $z$-scaling in $p p(p \bar{p})$ collisions for other particles sufficiently well. Note that the top-spectrum is in the limited kinematic region and the error bars of the data are large enough. We would like to stress that existing analyses were performed with $p_{T}$-distributions of the inclusive cross sections $E \mathrm{~d}^{3} \sigma / \mathrm{d} p^{3}$ which reveal strong dependence on the energy, angle, multiplicity and type of the produced particles. Based on the above comparison we have concluded that the Tevatron data on inclusive spectra of the top-quark production measured by the DØ Collaboration support the flavor independence of scaling function $\Psi(z)$ over the range of $z=0.02-2$. This result gives us an indication on self-similarity of top-quark production in $p \bar{p}$ collisions at $\sqrt{s}=1960 \mathrm{GeV}$.

The determination method of the momentum fractions allows us to analyze kinematics of the constituent interactions in the framework of the developed approach. Unlike other particles, the null value of $\varepsilon_{\text {top }}$ means that the energy loss of the top-quark production is zero or negligible. This result is expressed by condition $y_{a}=$ $y_{b}=1$ and by the value of recoil mass $M_{X}$ which is practically equal to the mass of the top-quark. The kinematics of the underlying sub-process is fully determined by momentum fractions $x_{1}$ and $x_{2}$ in this case. The fractions characterize the amount of the energy (momentum) of the incoming protons (antiprotons) carried by the interacting constituents which cause the inclusive particle production. In the general case of other particles, $x_{1}$ and $x_{2}$ are functions of $y_{a}$ and $y_{b}$ [15]. For the central interaction region, $x_{1}$ and $x_{2}$ are equal to each other. A comparison of fraction $x_{1}$ for the top-quark production with other heavy particles is shown in Figure 2. The illustration is presented as a function of the scaled transverse momentum $p_{T} / m$. One can see the $x_{1}$ growth with $p_{T} / m$. The value of $x_{1}$ is larger for the production of heavy particles as compared with the light ones. The exception for $J / \psi$ meson was observed (see discussed in [16]). It is a consequence of the relatively large value of $\varepsilon_{J / \psi} \approx 1$ related 


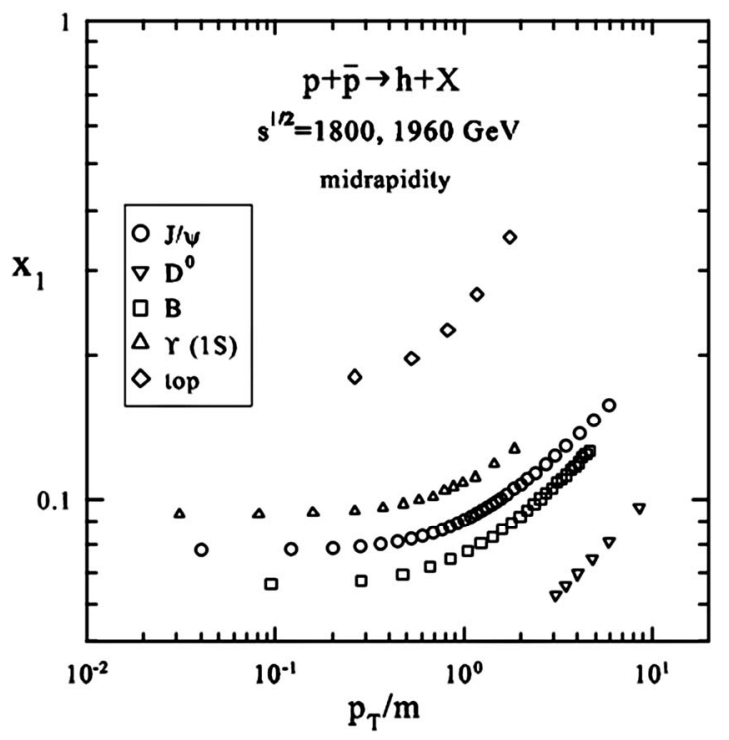

Figure 2. The momentum fraction $x_{1}$ as a function of the scaled transverse momentum $p_{T} / m$ of hadrons produced in $p \bar{p}$ collisions at $\sqrt{s}=1800$ and $1960 \mathrm{GeV}$ in the middle rapidity region.

with extra large energy dissipation in the final state accompanied by production of this particle. For a fixed value of $p_{T} / m$ the fraction $x_{1}$ decreases while increasing collision energy $\sqrt{s}$. The kinematic limit of the reaction $P_{1}+P_{2} \rightarrow p+X$ corresponds to $x_{1}=x_{2}=1$ at any collision energy and for any type of the inclusive particle.

\section{Conclusions}

We have presented the results of analysis of the data on inclusive spectra of the top-quark production in $p \bar{p}$ collisions at the energy $\sqrt{s}=1960 \mathrm{GeV}$ measured by the DØ Collaboration at the Tevatron. The transverse momentum spectra in $z$-presentation are compared with the data obtained for the heavy mesons $J / \psi, D^{0}, B$, and $Y$ at the Tevatron energies $\sqrt{s}=1960$ and $1800 \mathrm{GeV}$ in the central rapidity range. Based on the results presented here we conclude that the data on the transverse momentum distribution of the top-quark production in $p \bar{p}$ collision are in good agreement with flavor independence of the $z$-scaling. The result also supports the energy independence of the scaling function in the middle rapidity region. A tendency to $\Psi(z)$ saturation at low $z$ for the top-quark production is confirmed as well. The momentum fraction $x_{1}$ of the incoming protons for the top-quark was compared with the corresponding values for the heavy mesons measured at the Tevatron. Though production of the top-quark is characterized by no energy loss and constant recoil mass $M_{X} \approx m_{\text {top }}$, the fraction $x_{l}$ reveal similar dependences on the scaled transverse momentum $p_{T} / m$ as for the heavy mesons.
We assume that the data on the top-quark differential inclusive cross section over a wider range of $p_{T}$ and collision energy $\sqrt{s}$ at the Tevatron and LHC could be of interest to verify the flavor independence of $z$-scaling and self-similarity of top-quark production.

\section{Acknowledgements}

These investigations have been supported by the IRP AVOZ10480505, by the Ministry of Education of the Czech Republic grants LA08002, LA08015.

\section{REFERENCES}

[1] T. Affolder, et al., "Measurement of the Top Quark $\mathrm{p}_{\mathrm{T}}$ Distribution," Physical Review Letters, Vol. 87, No. 10, 2001, Article ID: 102001.

doi:10.1103/PhysRevLett.87.102001

[2] V. M. Abazov et al., "Dependence of the tbart Production Cross Section on the Transverse Momentum of the Top Quark," Physics Letters B, Vol. 693, No. 5, 2010, pp. 515-521. doi:10.1016/j.physletb.2010.09.011

[3] F. Abe, et al., "Observation of Top Quark Production in pbarp Collisions with Collider Detector at Fermilab," Physical Review Letters, Vol. 74, No. 14, 1995, pp. 26262631.

[4] S. Abachi et al., "Observation of the Top Quark," Physical Review Letters, Vol. 74, No.14, 1995, pp. 2632-2637.

[5] E. Laenen, "Top Physics: Theoretical Aspects," Physics at the LHC 2011, Perugia, 5-11 June 2011. http://www.pg.infn.it/plhc2011/index.html

[6] A. Garcia-Bellido, "Top Quark Physics at the Tevatron," Physics at LHC 2011, Perugia, 6-11 June, 2011. http://www.pg.infn.it/plhc2011/index.html

[7] Y. Peters, "Top Quark Properties," XXXI PHYSICS IN COLLISION, Vancouver, 28 August-1 September 2011. http://arxiv.org/abs/1112.0451

[8] E. Shabalina, "Particle Physics and Cosmology," 2010. http://confs.obspm.fr/Blois2010/index.html

[9] R. Kehoe, M. Narain, A. Kumar, "Review of Top Quark Physics Results," International Journal of Modern Physics A, Vol. 23, No. 3-4, 2008, pp. 353-470. doi:10.1142/S0217751X08039293

[10] W. Wagner, "Top Quark Physics in Hadron Collisions," Reports on Progress in Physics, Vol. 68, No. 10, 2005, pp. 2409-2494. doi:10.1088/0034-4885/68/10/R03

[11] A. Quadt, "Top Quark Physics at Hadron Colliders," European Physical Journal C, Vol. 48, No. 3, 2006, pp. 835-1000. doi:10.1140/epjc/s2006-02631-6

[12] E. W. N. Glover, et al., "Top Quark Physics at Colliders," Acta Physica Polonica B, Vol. 35, No. 11, 2004, pp. 2671-2694.

[13] I. Zborovský, Yu. Panebratsev, M. Tokarev and G. Škoro, "z-Scaling in Hadron-Hadron Collisions at High Energies," Physical Review D, Vol. 54, No. 9, 1996, pp. 55485557. doi:10.1103/PhysRevD.54.5548

[14] M. Tokarev, I. Zborovský, Yu. Panebratsev and G. Škoro, 
"A-Dependence of z-Scaling," International Journal of Modern Physics A, Vol. 16, No. 7, 2001, pp. 1281-1301. doi:10.1142/S0217751X01003391

[15] I. Zborovský and M. V. Tokarev, "Generalized z-Scaling in Proton-Proton Collisions at High Energies," Physical Review D, Vol. 75, No. 9, 2007, Article ID: 094008.

[16] I. Zborovský and M. V. Tokarev, "New Properties of zScaling: Flavor Independence and Saturation at Low z," International Journal of Modern Physics A, Vol. 24, No. 7, 2009, pp. 1417-1442. doi:10.1142/S0217751X09042992

[17] V. Khachatryan, et al., "Transverse Momentum and Pseudorapidity Distributions of Charged Hadrons in $p p$ Collisions at $\sqrt{s}=0.9$ and $2.36 \mathrm{TeV}$," Journal of High Energy Physics, Vol. 02, 2010, Article ID: 041.

[18] V. Khachatryan, et al., "Strange Particle Production in $p p$ Collisions at $\sqrt{s}=0.9$ and $7 \mathrm{TeV}$," Journal of High Energy Physics, Vol. 5, 2011, Article ID: 064.

[19] S. Chatrchyan, et al., "Measurement of the Inclusive Jet Cross Section in $p p$ Collisions at $\sqrt{s}=7 \mathrm{TeV}$," Physical Review Letters, Vol. 107, No. 13, 2011, Article ID: 132001 .

[20] J. Zhang (for ATLAS Collaboration), XIX International Workshop DIS2011, 11-15 April 2011, Newport News. http://conferences.jlab.org/DIS2011/

[21] M. V. Tokarev and I. Zborovský, "On Saturation of Charged hadron Production in $p p$ Collisions at LHC," Journal of Physics G: Nuclear and Particle Physics, Vol. 37, No. 8, 2010, Article ID: 085008. doi:10.1088/0954-3899/37/8/085008
[22] M. V. Tokarev and I. Zborovský, "Energy Loss in Hadron Production in $p p$ and Heavy Ion Collisions," Nuclear Physics B, Vol. 219-220, 2011, pp. 301-304. doi:10.1016/j.nuclphysbps.2011.10.116

[23] M. Tokarev and I. Zborovský, "First Test of z-Scaling in Hadron and Jet Production at LHC," XLI International Symposium on Multiparticle Dynamics (ISMD2011), Hiroshima, 26-30 September 2011. http://home.hiroshima-u.ac.jp/ismd2011/

[24] D. Acosta, et al., "Measurement of the $J / \psi$ meson and bhadron Production Cross Sections in p-barp Collisions at $\sqrt{s}=1960 \mathrm{GeV}, "$ Physical Review D, Vol. 71, 2005, Article ID: 032001. doi:10.1103/PhysRevD.71.032001

[25] D. Acosta, et al., "Measurement of Prompt Charm Meson Production Cross Sections in p-barp Collisions at $\sqrt{s}=$ 1:96 TeV," Physical Review Letters, Vol. 91, No. 24, 2003, Article ID: 241804. doi:10.1103/PhysRevLett.91.241804

[26] D. Acosta, et al., "Y Production and Polarization in p-bar p Collisions at $\sqrt{s}=1.8 \mathrm{TeV}$," Physical Review Letters, Vol. 88, No.16, 2002, Article ID: 161802. doi:10.1103/PhysRevLett.88.161802

[27] B. Alper, et al., "Production Spectra of $\pi^{ \pm}, K^{ \pm}, p^{ \pm}$at Large Angles in Proton-Proton Collisions in the CERN Intersecting Storage Rings," Nuclear Physics B, Vol. 100, No. 2, 1975, pp. 237-290. doi:10.1016/0550-3213(75)90618-5 